\title{
An Introduction to Drug Nomenclature
}

\author{
Indrani Devi Sarma ${ }^{1}$, Dhriti Kr Brahma ${ }^{2 *}$ and Himadri Sekhar \\ Dasgupta $^{1}$ \\ ${ }^{1} 2^{\text {nd }}$ year PGT, Department of Pharmacology, NEIGRIHMS, Shillong, India \\ ${ }^{2}$ Associate Professor, Department of Pharmacology, NEIGRIHMS, Shillong, India \\ *Corresponding Author: Dhriti Kr Brahma, Associate Professor, Department of \\ Pharmacology, NEIGRIHMS, Shillong, India.
}

Received: September 24, 2021

Published: October 22, 2021

(C) All rights are reserved by Dhriti Kr

Brahma., et al.

\begin{abstract}
The nomenclature of drugs is a complex process. A drug generally has three different categories of names from its discovery to the arrival in the market: chemical name, non-proprietary name and proprietary name. While chemical name is given when a new chemical entity (NCE) is developed and it describes the substance chemically, the non-proprietary name of a drug is the accepted name by a competent scientific body/authority. As the name suggests, the proprietary name or brand name is the name assigned by the manufacturer(s) and is the sole property or trade mark of the concerned pharmaceutical company developing it. The nonproprietary names of newer drugs are kept uniform by an agreement to use the Recommended International Nonproprietary Name (rINN) in all member countries of World Health Organization (WHO). The system of INN of drugs was initiated by WHO and began operating in 1953. The crux of the non-proprietary naming system is the collection of short name fragments called stems. They define the pharmacologically related group to which the INN belongs. The WHO uses its stem classification system in INN Programme by following a core list to categorize the main activity of pharmaceutical substances. Each category included in the list has an appropriate code consisting of a capital letter and three digits, category, specific stem and appropriate information..
\end{abstract}

Keywords: Chemical Name; Non-proprietary Name; Proprietary Name; INN System

\section{Introduction}

The term drug nomenclature implies to the systematic and scientific naming of drugs. A drug generally has three different categories of names: chemical name, non-proprietary name and proprietary name [1].

A chemical name is given when a new chemical entity (NCE) is developed and it describes the substance chemically, e.g. 7-chloro-1,3-dihydro-1-methyl-5-phenyl-2H-1,4-benzodiazepin- 2-one for diazepam. It is the name given to drug in accordance with rules of chemical nomenclature established by International Union of Pure and Applied Chemistry (IUPAC) [2]. It is useful for chemists or technical personals as it provides the precise arrangement of atoms and atomic groups in the molecule. It is cumbersome and not used to identify the drug in a clinical (prescribing) or marketing situation. A code name, e,g. R015-1788 (later named flumazenil) is the name given by the manufacturer for convenience and simplicity before an approved name.

A non-proprietary name of a drug, after its regulatory approval, is the accepted name by a competent scientific body/authority e.g. British Approved Name (BAN), Japanese Accepted Name (JAN) and United States Adopted Name (USAN) etc. [3,4]. The non-proprietary names of newer drugs are kept uniform by an agreement to use the Recommended International Nonproprietary Name (rINN) in all member countries of World Health Organization (WHO) [5].

A proprietary name or brand name is the name assigned by the manufacturer(s) and is the property or trade mark of the concerned pharmaceutical farm. Thus a single drug may be sold under 
different proprietary names by different manufacturers. For example, haloperidol is marketed under the brand names of Bezydol-P, Brain-Rest, Cizoren, Depidol, Gendol and Dolcin etc.

The INN name or more popularly known as generic name identifies a pharmaceutical substance by a unique name that is globally recognized to varying extent and is public property. We will be restricting our discussion on the different aspects of INN nomenclature.

\section{The INN nomenclature system}

The system of INN was initiated by WHO through the World Health Assembly resolution

WHA3.11 and began operating in 1953 [6]. The first list of international Non-proprietary Names for pharmaceutical substances was published in the same year. Since then, thousands of pharmaceutical substances have been designated as INNs so far and continuously updated. WHO works in close collaboration with the major national nomenclature commissions e.g. BAN British Approved name, JAN Japanese Accepted Name, USAN United States Adopted Name etc.). An International Nonproprietary Name (INN) identifies a pharmaceutical substance by a unique name that is globally recognized and is public property. INNs help to reduce confusion in drug nomenclature and allow us an understanding of the drug even when that individual drug is not known. They are the vital piece of information that is compulsory on medicine labels. INNs are nonproprietary in nature, which implies that the same INN can be used by all manufacturers of that pharmaceutical substance, irrespective of the brand name under which the drug is marketed by each manufacturer. These names are intended to be used in pharmacopoeias, labelling, advertising, drug regulation and scientific literature. The term approved name is used until the drug is included in an official pharmacopoeia. The approved name becomes official name after its appearance in the official pharmacopoeia.

\section{The procedure of INN selection}

Any person or company proposing and requesting for an INN needs to apply to WHO online at http://www.who.int/medicines/ services/inn/en/index.html. Applications are also accepted by WHO made through the national nomenclature committee from the applicants where national nomenclature commissions exist. Members of the WHO expert panel on International Pharmacopoeia and Pharmaceutical Preparations or sometimes other panel examine with a formal procedure and select a nonproprieatary, agreed name based on the information provided. A four month window period is kept thereafter for any comments or formal objection to the proposed name by any person. If no objection is raised within that period, this agreed name is published as the recommended INN [7]. WHO has strictly recommended in 1993 that pharmaceutical companies should not derive trade names from INNs as such practice may frustrate the rational selection of INNs and compromise the patient safety by confusing the drug nomenclature. In India, the trademark registration of words that are declared as INNs or those that are deceptively similar to INNs is prohibited under Section 13 (b) of the Trade Marks Act, 1999.

\section{INN stems}

The root of the non-proprietary naming system is the collection of short name fragments called stems [8].

They define the pharmacologically related group to which the INN belongs. Each stem has a meaning connected to a particular drug class or mode of action. The stems and their definitions have been selected by WHO experts and are used when selecting new international nonproprietary names. The naming process itself "is an evolving type of science and therefore the nomenclature process is ongoing and constantly under revision. Definitions of older stems are modified as and when newer information becomes available. An INN generally includes the "common stem" expressing the pharmacologically-related group to which the substance belongs. Names conveying anatomical, physiological, pathological or therapeutic suggestions are avoided while devising an INN. International connotations are also considered while selecting an INN. A name that sounds perfectly fine in English might have bad or even obscene connotations elsewhere. Therefore, to make pronunciation possible in various languages, the letters " $\mathrm{h}$ " and " $\mathrm{k}$ " are avoided; " $e$ " is used instead of "ae" and "oe", "i" instead of " $y$ ", " $t$ " instead of "th" and "f" instead of "ph". Stems are generally used as suffixes but sometimes they are also used as prefixes.

\section{Classification system used by the INN Programme}

The WHO stem classification system used by the INN Programme follows a core list to categorize the main activity of pharmaceutical substances while devising an INN. Each category included in the list has an appropriate code consisting of a capital letter and three digits, category, specific stem and appropriate information. When INNs for substances belonging to a given category include a specific stem, appropriate information is included in the list. 
However, different stems may exist under the same code and category.

\section{Conclusion}

Today, due to the existence of this system of naming, it is rare that a national generic name for a new pharmaceutical substance is different from the INN. The non-proprietary names of many older drugs now have been modified to be commensurate with rINN. But, it is difficult to do away with some of the well establish old names e.g. lignocaine and lidocaine. The pharmaceutical industry is a rapidly growing one as the number of drug substances being registered is constantly increasing. It requires a vibrant and strong nomenclature system to ensure the identification of each pharmaceutical compound by a unique, universally available and accepted name. The international nomenclature system for pharmaceutical products is crucial for the clear identification, safe prescription and dispensing of medicines to patients, and for communication and exchange of information among health professionals and scientists worldwide. However, challenges do exist till today in global implementation of the resolution in relation to INNs. For India, Section 13 (B) of the Trade Mark Act, 1999 prohibits the registration of names of chemical elements or INNn which have been declared by the WHO and notified by the Registrar of Trade Marks. Nevertheless, there were reported incidents of marketing of drugs under the names that are similar to INNs.

\section{Bibliography}

1. Gundersen L. "The complex process of naming drugs". Annals of Internal Medicine 129 (1998): 677-678.

2. The Commission on the Nomenclature of Organic Chemistry (1971) [1958 (A: Hydrocarbons, and B: Fundamental Heterocyclic Systems), 1965 (C: Characteristic Groups)]. Nomenclature of Organic Chemistry (3rd edition combined ed.). London: Butterworths.

3. United States Adopted Names Council. Naming guidelines: rules for coining names. American Medical Association.

4. British Approved Names: Guiding Principles (2006).

5. WHO, Guidance on INN.

6. WHO, Guidelines on the Use of International Nonproprietary
Names (INNs) for Pharmaceutical Substances (Geneva: World Health Organization) (1997): 13.

7. WHO, Procedure for the Selection of Recommended International Nonproprietary Names for Pharmaceutical Substances, text adopted by Executive Board of WHO in Resolution EB 15.R7, 1955 and amended by Resolution EB 43.R9, 1969, Article 2, reprinted in WHO Drug Information 19.2 (2005): 189190.

8. The use of stems in the selection of International Nonproprietary Names (INN) for pharmaceutical substances" (PDF). World Health Organization. 2011. WHO/EMP/QSM/2011.

\section{Volume 5 Issue 11 November 2021 (C)All rights are reserved by Dhriti Kr Brahma., et al.}

